\title{
Research on the Numerical Simulation of the Nonlinear Dynamics of a Supercavitating Vehicle
}

\author{
Tianhong Xiong, ${ }^{1}$ Xianyi $\mathrm{Li}^{2}{ }^{2}$ Yipin $\mathrm{Lv},{ }^{1}$ and Wenjun $\mathrm{Yi}^{1}$ \\ ${ }^{1}$ National Key Laboratory of Transient Physics, Nanjing University of Science and Technology, Nanjing 210094, China \\ ${ }^{2}$ College of Mathematical Science, Yangzhou University, Yangzhou 225002, China \\ Correspondence should be addressed to Xianyi Li; mathxyli@yzu.edu.cn
}

Received 27 April 2016; Accepted 21 July 2016

Academic Editor: Dumitru I. Caruntu

Copyright (c) 2016 Tianhong Xiong et al. This is an open access article distributed under the Creative Commons Attribution License, which permits unrestricted use, distribution, and reproduction in any medium, provided the original work is properly cited.

\begin{abstract}
Little is known about the movement characteristics of the supercavitating vehicle navigating underwater. In this paper, based on a four-dimensional dynamical system of this vehicle, its complicated dynamical behaviors were analyzed in detail by numerical simulation, according to the phase trajectory diagram, the bifurcation diagram, and the Lyapunov exponential spectrum. The influence of control parameters (such as various cavitation numbers and fin deflection angles) on the movement characteristics of the supercavitating vehicle was mainly studied. When the system parameters vary, various complicated physical phenomena, such as Hopf bifurcation, periodic bifurcation, or chaos, can be observed. Most importantly, it was found that the parameter range of the vehicle in a stable movement state can be effectively determined by a two-dimensional bifurcation diagram and that the behavior of the vehicle in the supercavity can be controlled by selecting appropriate control parameters to ensure stable navigation.
\end{abstract}

\section{Introduction}

Liquid vaporization of a liquid occurs at any point when the pressure at that point is reduced below a critical value. In the initial stage, the above phenomenon is microscopic. As time progresses macroscopically, small bubbles arise. Further, these macroscopic bubbles join to form larger cavum of steam and gas in the interface of the liquid or liquid and solid, known as cavities [1]. The emergence, development, and crumble processes of the cavity are called cavitation phenomena. Supercavitation is a state in which the cavity appears on the whole surface of the object and in the liquid near the end. In this state, the formed cavity is like a big steam bag, exceeding the end of the object or loading the entire object inside; hence the name is supercavity [1-3].

A dimensionless cavitation number $\sigma$ that reflects the cavity is introduced to investigate the characteristic of the supercavity in a general case. The cavitation number $\sigma$ is defined as $\sigma=\left(P_{\infty}-P_{c}\right) / 0.5 \rho V^{2}$, where $P_{\infty}$ is the ambient pressure, $P_{c}$ is the cavity pressure, $\rho$ is the water density, and $V$ is the vehicle velocity [1]. Once the supercavity becomes stable, most of the vehicle's surface is surrounded by gases and the resistance of the vehicle decreases sharply. This increases the navigation velocity and the distance travelled by the vehicle [2-4]. However, when a supercavitation vehicle is navigating at high speed under the water, most of the vehicle is surrounded by the cavity; the wet area will be significantly reduced, which results in the loss of most of the buoyancy. The parts of the vehicle that are in contact with water are mainly a cavitator in the front and fins at the rear of the vehicle. When coming into contact with the cavity wall, the fin will produce complex nonlinear planing force, which will increase the frictional resistance of the vehicle, causing vibrations and impact to the vehicle [5-10]. Hence, the key to ensuring stable underwater navigation of the vehicle lies in effectively controlling the behavior of the supercavitation vehicle and reducing the impact of the collision between the vehicle and the cavity wall.

Based on a four-dimensional dynamical system of a supercavitating vehicle, its complicated physical phenomena were studied by means of multiple dynamical analysis, aiming at the complex nonlinear planing force, generated by the 
contact between the fins and the cavity walls. The most important finding of this study was that the region and parameter range of the vehicle in a stable movement state are determined by the two-dimensional bifurcation diagram. The movement characteristics of the supercavitation vehicle under different control parameters were also discussed in detail.

To the best of our knowledge, it is very difficult to find any related work in this paper up till now.

\section{Dynamic Modeling of the Supercavitating Vehicle}

2.1. Force Analysis. When the underwater vehicle is navigating at high speed in the supercavitating state, most of the vehicle will be enveloped by the cavity and only a small part of the surface will have a contact with the water. While the cavitator at the front has a direct contact with water, the cavitator can rotate by a certain angle. Different hydrodynamic power can be provided for the vehicle with the change of the angle, and a planing force can be produced when the fin contacts with the cavity wall. Four fins were symmetrically arranged in the rear part of the vehicle. A part of the fin penetrates the cavity wall to have a direct contact with the water, thus providing the required force and momentum to stabilize and control the vehicle with the cavitator. In this case, the control surface is composed of the cavitator and the four fins [5]. The deflection angles of the cavitator and the fins are usually selected as the feedback control inputs to ensure the stable underwater movement of the vehicle. The shape and the force diagram of the supercavitating vehicle are presented in Figure 1.

The forces acting on the vehicle in its own coordinate system are indicated in Figure 1. The main forces include the lift force on the cavitator, $F_{\text {cavitator }}$, the lift force on the fin, $F_{\text {fins }}$, the gravity at the centroid of the vehicle, $F_{\text {gravity, }}$ and the planing force generated by the interaction between the fin and the cavity wall, $F_{\text {planing }}$, the last of which is a complicated nonlinear planing force that consequently causes vibration and impact to the vehicle. The expression of the planing force is as follows [11]:

$$
F_{\text {planing }}=-V^{2}\left[1-\left(\frac{R^{\prime}}{h^{\prime}+R^{\prime}}\right)^{2}\right]\left(\frac{1+h^{\prime}}{1+2 h^{\prime}}\right) \alpha,
$$

where $V$ is the velocity of the vehicle and $R^{\prime}=\left(R_{c}-\right.$ $R) / R$, where $R_{c}$ and $R$ are the radius of the cavity and the vehicle, respectively. The immersion depth of the aft of the supercavitating vehicle $h^{\prime}$ is given as follows [11]:

$$
h^{\prime}=\tanh (k w) \frac{L}{2 R V} f(w),
$$

where

$$
\begin{gathered}
f(w)=2 w+\left(w+w_{t 0}\right) \tanh \left[-k\left(w+w_{t 0}\right)\right] \\
+\left(w-w_{t 0}\right) \tanh \left[k\left(w-w_{t 0}\right)\right],
\end{gathered}
$$

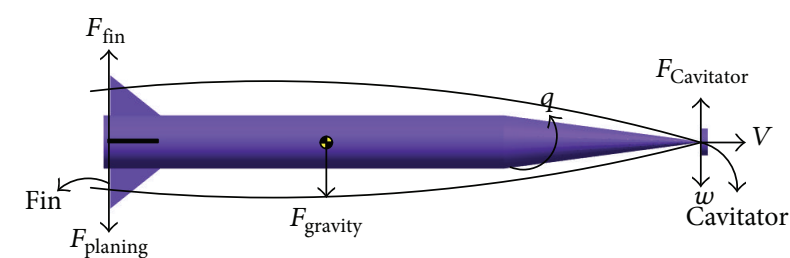

FIGURE 1: Shape and force diagram of the supercavitating vehicle.

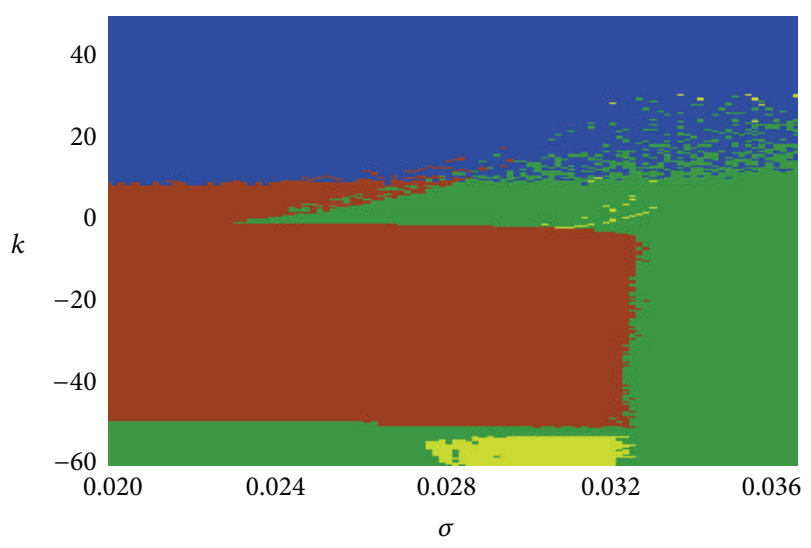

FIgURE 2: Dynamic behavior distribution diagram of the system.

where the positive value of $w$ at the transition point is $w_{t 0}=\left(R_{c}-R\right) V / L$ and $k$ is a constant used to control the approximated error, which is generally set to 300 .

The geometrical angle between the vehicle centerline and the cavity centerline is the immersion angle $\alpha$ of the supercavitating vehicle, expressed as [11]

$$
\alpha=\frac{w}{V}-\tanh (k w) \frac{\dot{R}_{c}}{V},
$$

where $R_{c}$ is the cavity radius and $\dot{R}_{c}$ is the shrinkage ratio at a distance $L$ from the cavitator.

2.2. Dynamic Modeling. Through the interactive relationship between the vehicle and the cavity, obtained in the previous section, the model can be established based on the force equations [12]. According to the coordinate system presented in [13], the origin is the center of the disk cavitator at the front of the supercavitating vehicle. $X$-axis aligns with the symmetry axis of the vehicle and points forward. $Z$-axis is perpendicular to $X$-axis, facing vertically downward, and $w$ is the velocity in $Z$-axis direction. $V$ represents the longitudinal velocity of the cavitator at the vehicle front, and $\theta, q$, and $z$ are the pitching angle, pitching rate, and depth of the vehicle, respectively. 


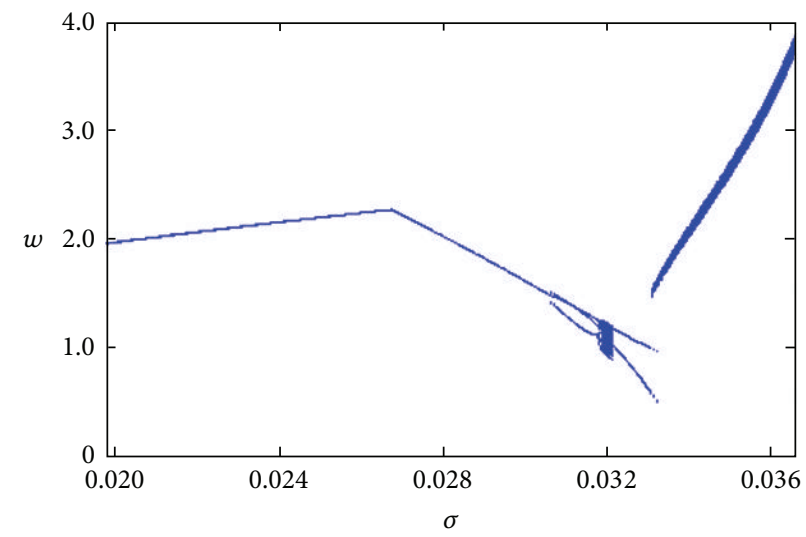

FIgURE 3: Bifurcation diagram varying with $\sigma$ when $k=1$.

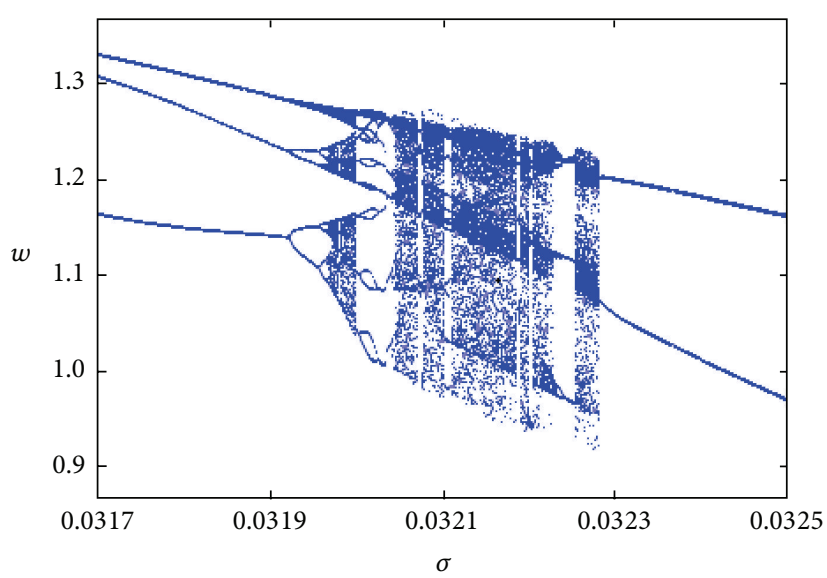

Figure 4: Bifurcation diagram for $0.0315<\sigma<0.0325$.

According to the theory of rigid body dynamics, the following relationship can be derived, relating the above variables [13]:

$$
\begin{aligned}
\left(\begin{array}{c}
\dot{z} \\
\dot{w} \\
\dot{\theta} \\
\dot{q}
\end{array}\right)= & \left(\begin{array}{cccc}
0 & 1 & -V & 0 \\
0 & a_{22} & 0 & a_{24} \\
0 & 0 & 0 & 1 \\
0 & a_{42} & 0 & a_{44}
\end{array}\right)\left(\begin{array}{l}
z \\
w \\
\theta \\
q
\end{array}\right) \\
& +\left(\begin{array}{cc}
0 & 0 \\
b_{21} & b_{22} \\
0 & 0 \\
b_{41} & b_{42}
\end{array}\right)\left(\begin{array}{l}
\delta_{e} \\
\delta_{c}
\end{array}\right)+\left(\begin{array}{l}
0 \\
c_{2} \\
0 \\
0 \\
0 \\
d_{2} \\
0 \\
d_{4}
\end{array}\right)
\end{aligned}
$$

where

$$
\begin{aligned}
& a_{22}=\frac{C V T}{m}\left(\frac{-1-n}{L}\right) S+\frac{17}{36} n L, \\
& a_{24}=V T S\left(C \frac{-n}{m}+\frac{7}{9}\right)-V T\left(C \frac{-n}{m}+\frac{17}{36}\right) \frac{17}{36} L^{2}, \\
& a_{42}=\frac{C V T}{m}\left(\frac{17}{36}-\frac{11 n}{36}\right), \\
& a_{44}=\frac{-11 C V T n L}{36 m} b_{21}=\frac{C V^{2} T n}{m}\left(\frac{-S}{L}+\frac{17 L}{36}\right), \\
& b_{22}=\frac{-C V^{2} T S}{m L}, \\
& b_{41}=\frac{-11 C V^{2} T n}{36 m} \\
& b_{42}=\frac{17 C V^{2} T}{36 m} \\
& c_{2}=g
\end{aligned}
$$




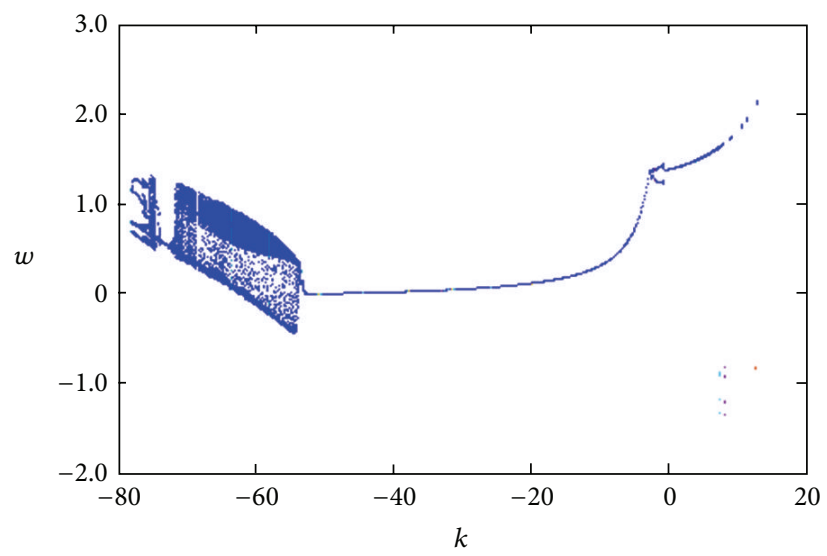

FIGURE 5: Bifurcation diagram varying with $k$ when $\sigma=0.0315$.

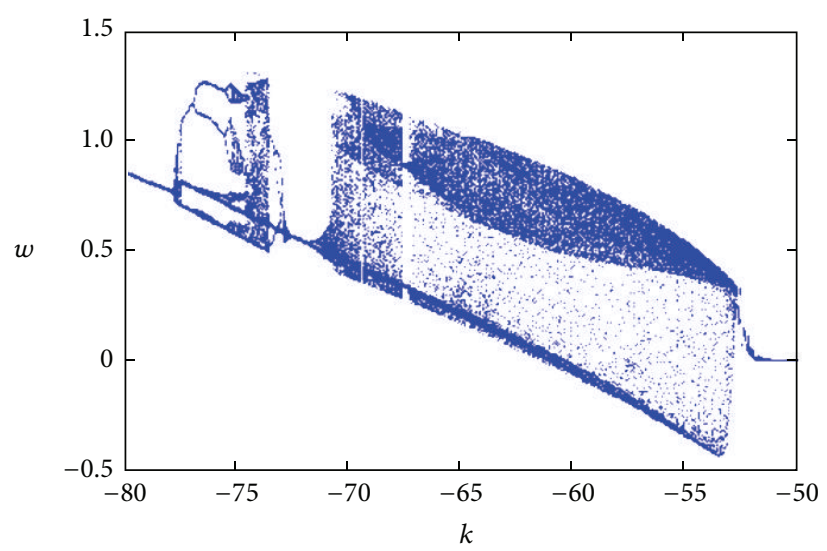

FigURE 6: Bifurcation diagram for $-80<k<-50$.

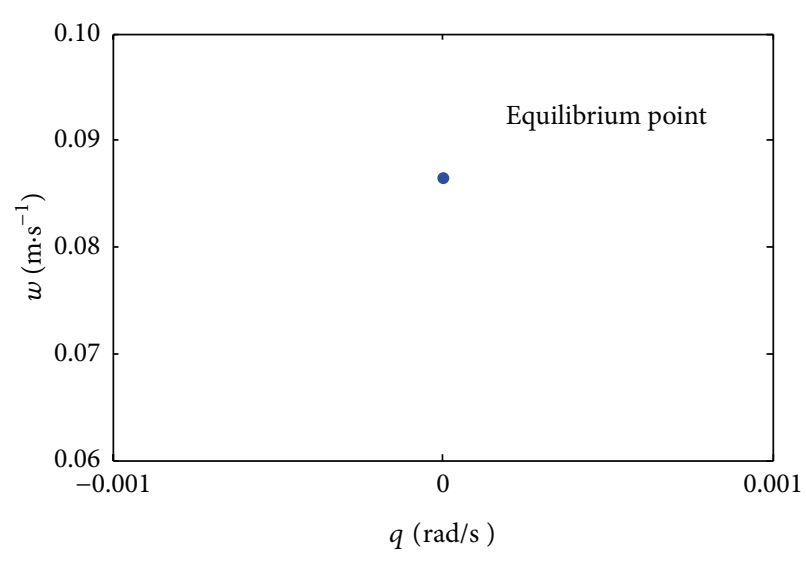

(a)

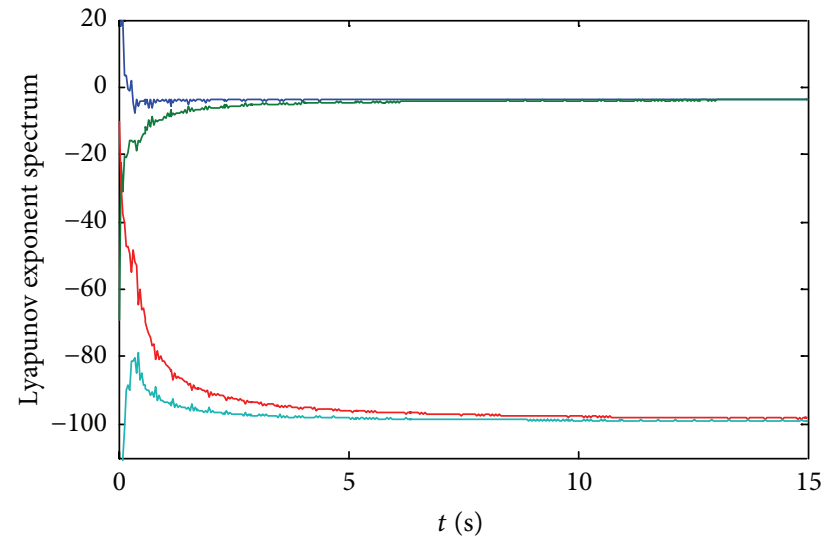

(b)

Figure 7: (a) Phase trajectory diagram in $w-\theta$ plane and (b) Lyapunov exponent spectrum.

$$
\begin{aligned}
d_{2} & =\frac{T}{m}\left(\frac{-17 L}{36}+\frac{S}{L}\right), \\
d_{4} & =\frac{11 T}{36 m} \\
S & =\frac{11}{60} R^{2}+\frac{133 L^{2}}{405},
\end{aligned}
$$$$
T=\frac{1}{7 S / 9-289 L^{2} / 1296}
$$ 


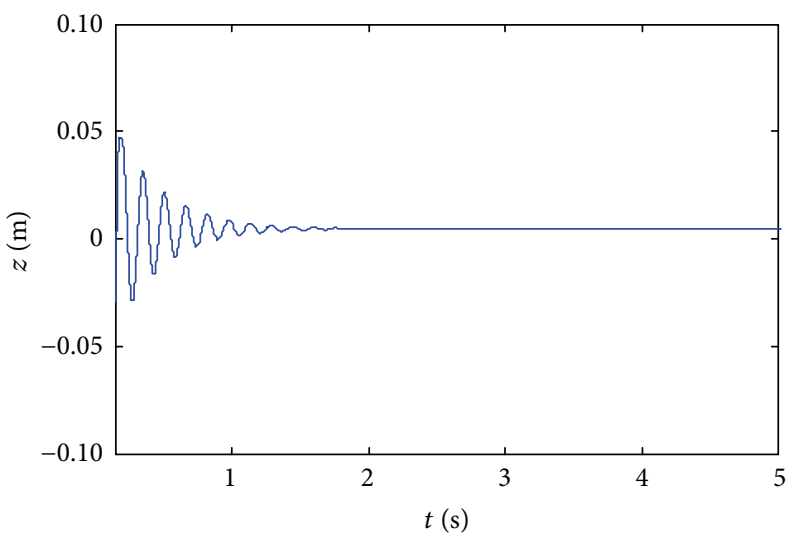

(a) Vertical position

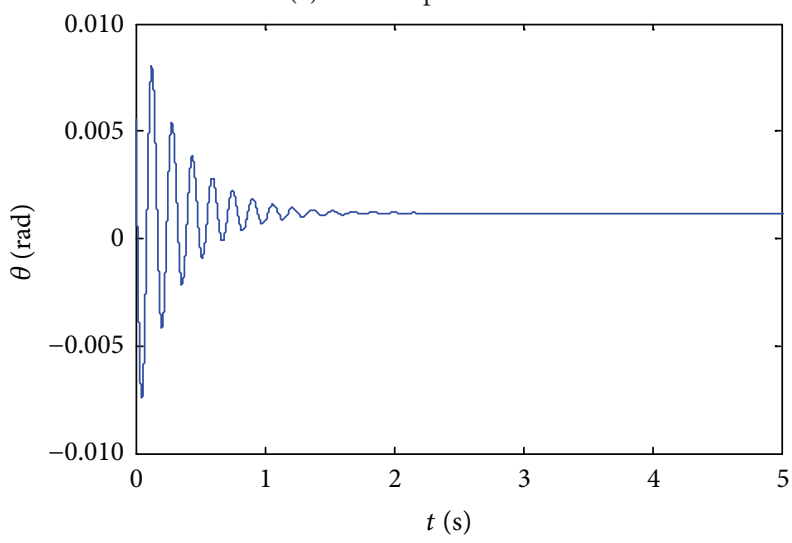

(c) Pitch angle

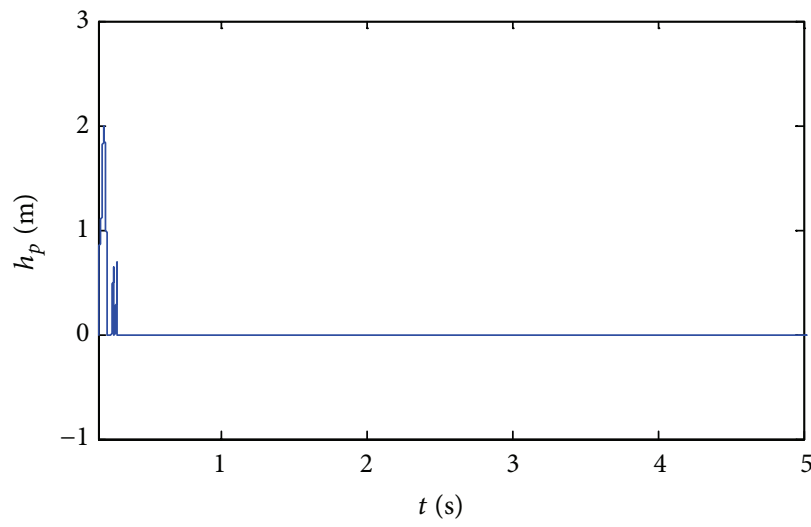

(e) Immersion depth

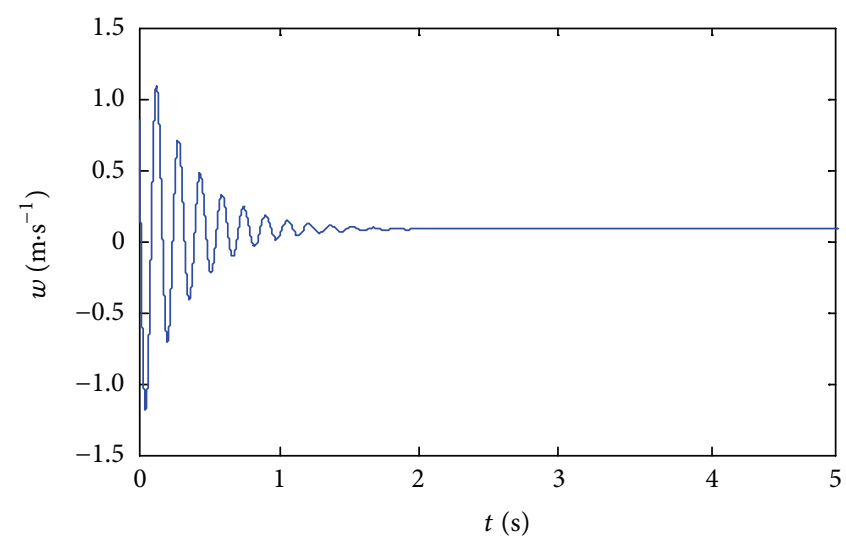

(b) Transverse speed

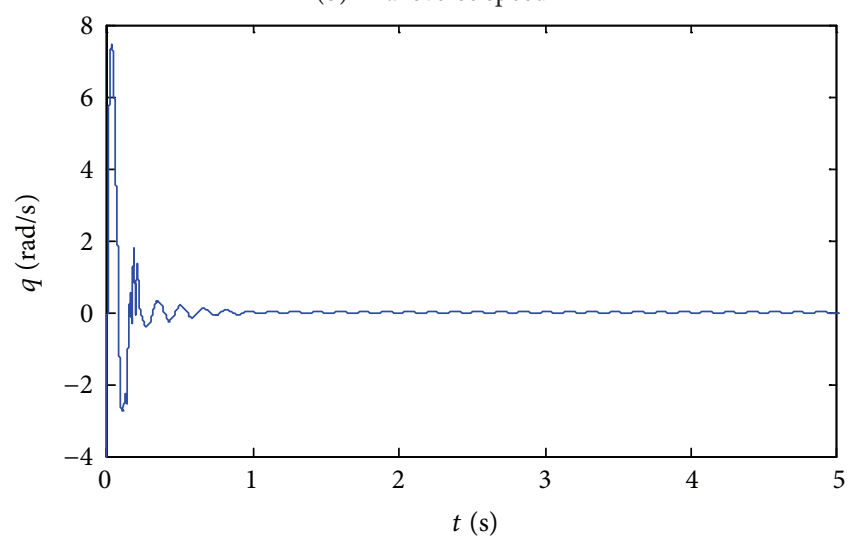

(d) Pitch rate

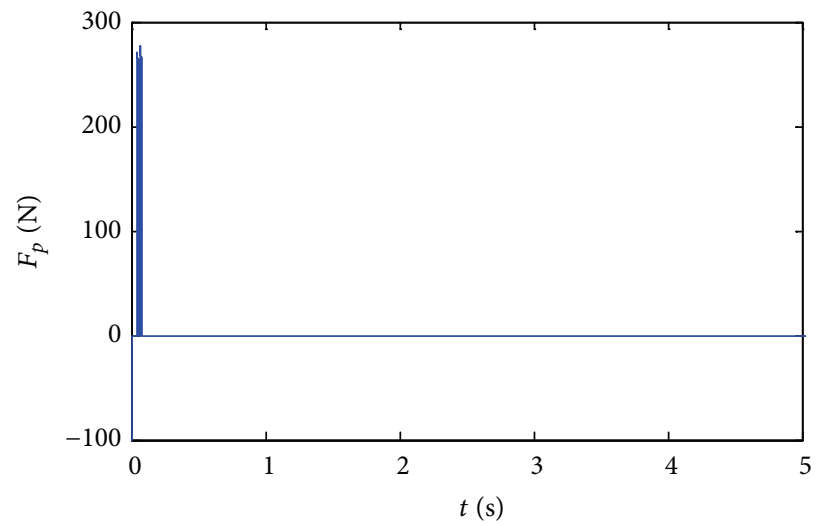

(f) Planing force

FIgURE 8: The motion state of the system when $k=-21.95$.

The feedback controller is designed for the supercavitating vehicle with control inputs being the deflection $\delta_{e}$ of the fin and the deflection $\delta_{c}$ of the cavitator. $\delta_{e}=k z$ and $\delta_{c}=15 z-30 \theta-0.3 q[12,14]$ were adopted in this paper, where $k$ is the feedback gain of the control variable $z$.

\section{Dynamic Behavior of the Underwater Supercavitating Vehicle}

According to [15], the system parameters of the supercavitation vehicle are as follows: $g=9.81 \mathrm{~m} / \mathrm{s}^{2}, m=2, R_{n}=$
$0.0191 \mathrm{~m}, R=0.0508 \mathrm{~m}, L=1.8 \mathrm{~m}, V \in[67.7,92.3] \mathrm{m} / \mathrm{s}$, $\sigma \in[0.0198,0.0368], n=0.5$, and $C_{x 0}=0.82$. To realize the stable movement of the supercavitating vehicle, the effects of cavitation number $\sigma$ and the fin control law $k$ on the stable movement state of the vehicle were analyzed based on the four-dimensional dynamical system. Here, the rest of the parameters remain constant and the two-dimensional bifurcation diagram $(\sigma, k)$ is presented in Figure 2, where the parameters are the cavitation number $\sigma$ and the control gain $k$ of the fin deflection angle $\delta_{e}$. 


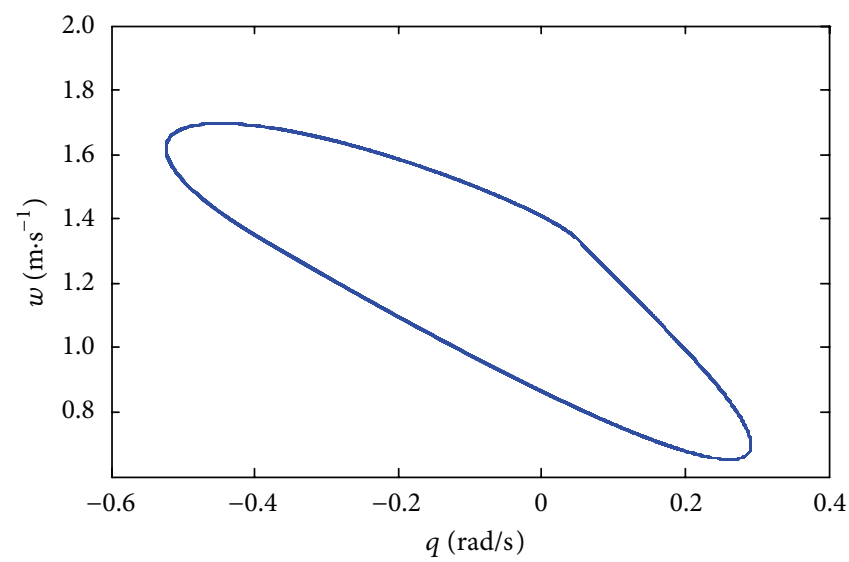

(a)

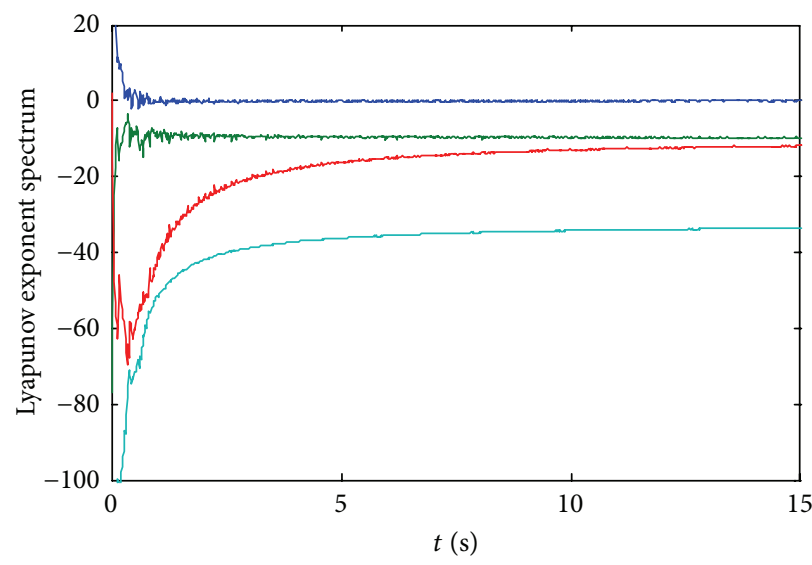

(b)

Figure 9: (a) Phase trajectory diagram in $w-\theta$ plane when $k=5$ and (b) Lyapunov exponent spectrum.

In the phase space of $(\sigma, k)$, the dynamic behavior of the system is presented in Figure 2. The horizontal section is the bifurcation diagram of the system for different cavitation numbers $\sigma$ and the vertical section is the bifurcation diagram of the system when the control gain $k$ varies. The parameter ranges for different system states can be determined by the two-dimensional bifurcation diagram. The region in red represents the stable movement state of the vehicle, which means the vehicle will navigate steadily when $\sigma$ and $k$ are equal to the values corresponding to any point $(\sigma, k)$ within this region. The green area shows the periodic oscillatory nature of the vehicle movement, which means that the vehicle will oscillate periodically and, hence, will become unstable. Moreover, the vehicle navigating with the states of the yellow area will suffer from vibration and impact and then collapse. When the vehicle alters from the steady state to the periodic state, the Hopf bifurcation occurs. The boundary between the red and green regions, that is, the critical switching line of the stable state and the periodic state, is also called the Hopf bifurcation line. Similarly, the boundary between the green and yellow areas indicates the switch between the periodic and chaotic states, where the physical phenomena, such as tangent bifurcation and period doubling bifurcation, can occur.

It can be observed from (1) that, in the four-dimensional dynamical system of the underwater vehicle, only the planing force $F_{\text {planing }}$ is the nonlinear force associated with the system state variable and the vertical velocity $w$. This is primarily attributed to the fact that the complicated nonlinear force acts on the fin of the vehicle that the vehicle suffers from vibration, impact, and even collapse due to unstable movement. Therefore, the nonlinear dynamic characteristics can be further understood by analyzing the system from the point of view of nonlinearity, thus preparing for the stable control of the supercavitating vehicle.

3.1. Nonlinear Dynamical Characteristic of the Vehicle under Different Cavitation Values. According to the dynamical behavior distribution diagram, presented in Figure 2, the bifurcation diagram between the system state variable $w$ and the cavitation number $\sigma$ is provided in Figure $3(k=1$, i.e., $\delta_{e}=z$ and $\left.\delta_{c}=15 z-30 \theta-0.3 q\right)$. Some simple explanations are given as follows.

When the cavitation number $\sigma$ of the system falls in the range of $[0.0198,0.02687]$, the trajectory of the vehicle converges to a stable equilibrium point.

When $\sigma$ is equal to 0.02687 , the Hopf bifurcation occurs, as a result of which the stable equilibrium point becomes the stable periodic trajectory and the vehicle oscillates periodically.

After a series of period doubling bifurcation, the system falls into a chaotic state and the vehicle suffers from huge impact. When the cavitation number $\sigma$ is approximately 0.03083 , the system has three stable periodic trajectories.

The bifurcation diagram shown in Figure 3, when $\sigma \in$ $[0.0315,0.0325]$, is magnified in Figure 4, which depicts the diversified bifurcation behaviors of the system.

After a series of period doubling bifurcations, the system shifts from three periodic trajectories into three huge chaotic attractors, respectively. when $\sigma$ is approximately 0.03197 , this phenomenon is referred to as chaos crisis [12].

When $\sigma$ is equal to 0.032 , the chaotic attractors suddenly change into periodic trajectories and form one period-2 window and two period-3 windows. This phenomenon is referred to as tangent bifurcation. The tangent bifurcation will cause intermittent chaos and the periodic trajectories suddenly develop chaotic bands in the periodic window after experiencing a period doubling bifurcation.

When $\sigma$ is equal to 0.03204 the secondary chaotic band coincides with the unstable periodic trajectories, which then causes the chaotic crisis. The secondary narrow chaotic band will then transform into a broad chaotic band.

With the increase of $\sigma$, the obvious period-2 window occurs for $\sigma \in[0.03207,0.03225]$ and when $\sigma$ is approximately 0.032228 , the broad chaotic band suddenly changes into two periodic trajectories.

3.2. Nonlinear Dynamic Characteristic of the Vehicle under Different Fin Deflection Angles. When the cavitation number 


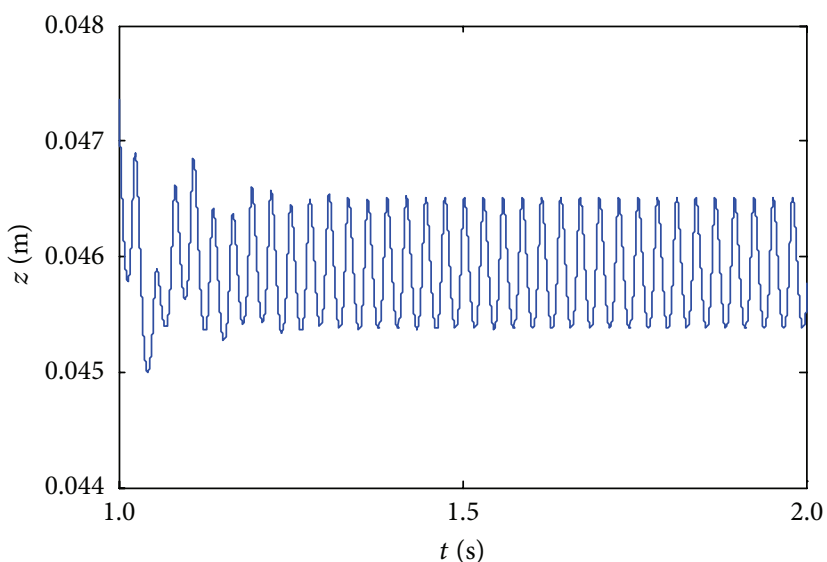

(a) Vertical position

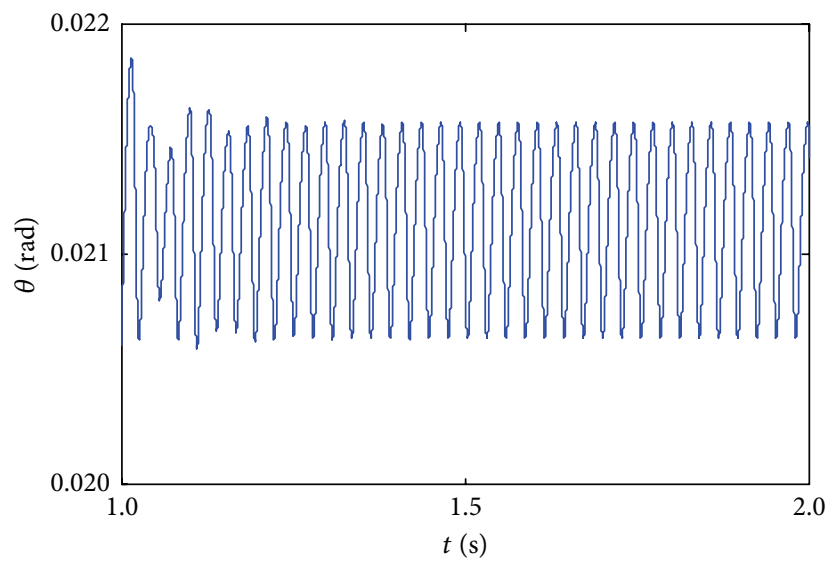

(c) Pitch angle

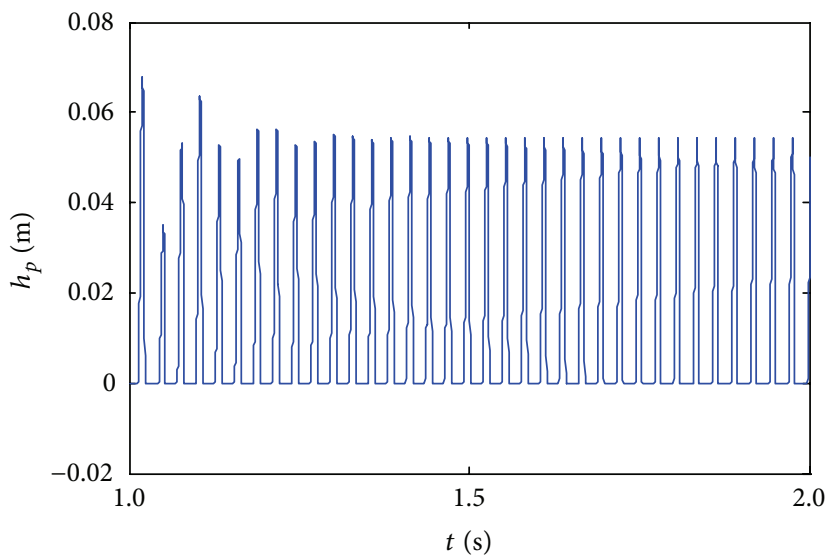

(e) Immersion depth

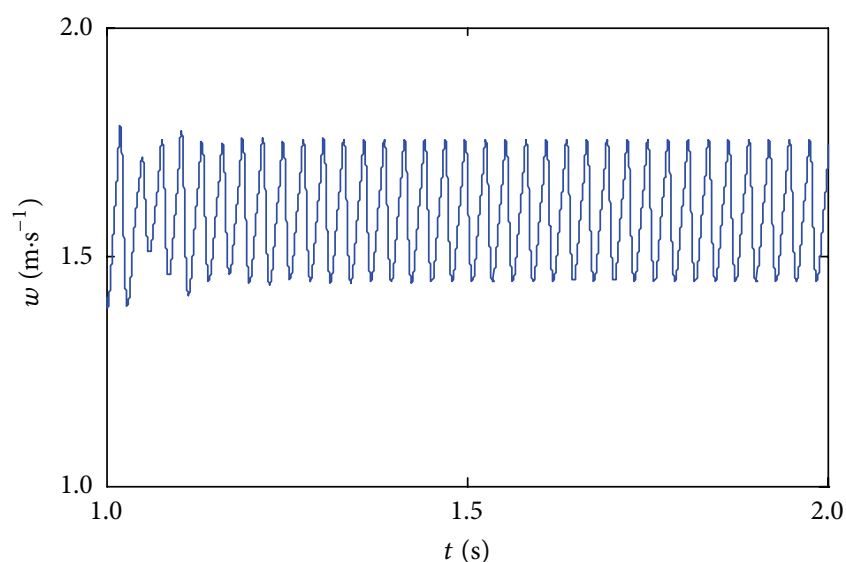

(b) Transverse speed

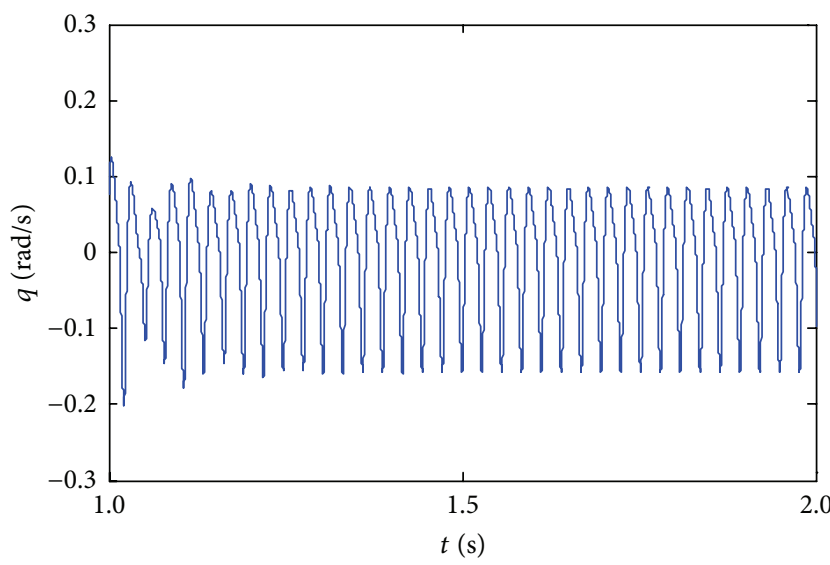

(d) Pitch rate

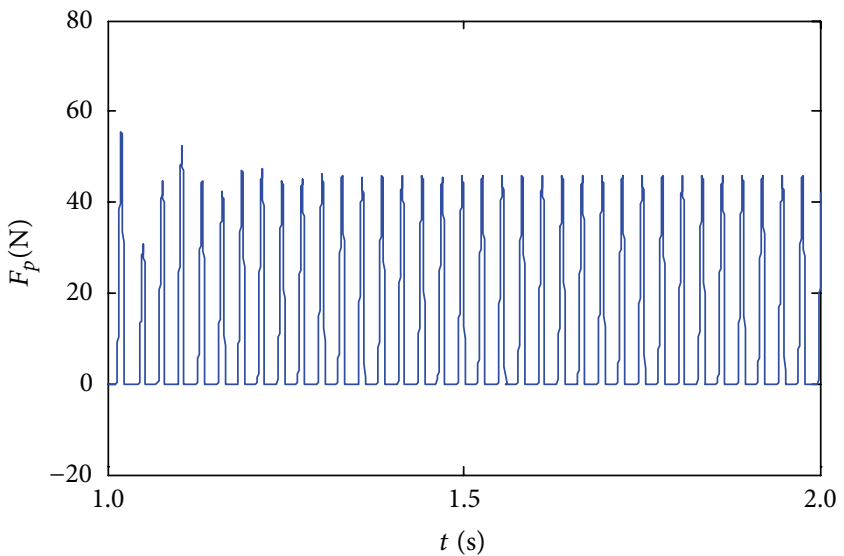

(f) Planing force

Figure 10: The motion state of the system when $k=5$.

is set as $\sigma=0.0315$, the cavitator deflection angle is $\delta_{c}=$ $15 z-30 \theta-0.3 q$ and the fin deflection angle is $\delta_{e}=k z$. The bifurcation diagram of the supercavitating vehicle, between the system state variable $w$ and the control gain $k$ of the fin deflection angle, is presented in Figure 5. According to the dynamical behavior distribution presented in Figure 2, the effective range of the control gain $k$ for the supercavitating vehicle is presented in Figure 5 when $\sigma=0.0315$.

Figure 5 shows that when $k$ falls within the wider range $[-76.14,-52.38]$, the system is in a chaotic state and finally the period-2 trajectory occurs through period doubling bifurcation. 


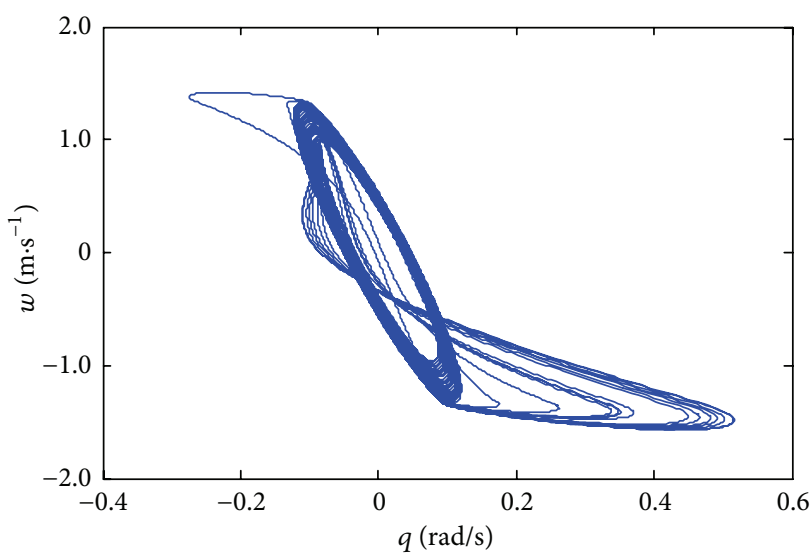

(a)

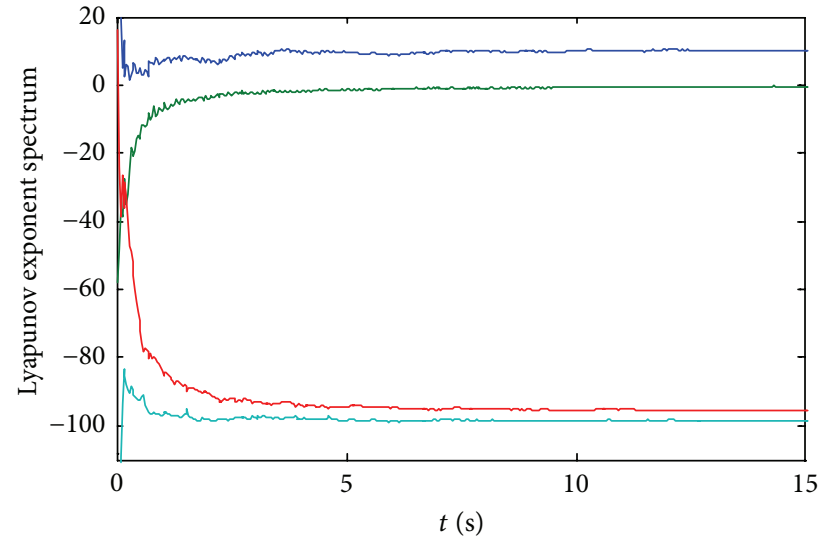

(b)

FIgURE 11: (a) Phase trajectory diagram in $w-\theta$ plane when $k=-55$ and (b) Lyapunov exponent spectrum.

When $k$ is approximately 8.56 , the periodic state ends and the system is in the divergent state. The corresponding magnified part in Figure 5 for $-80<k<-50$ is given in Figure 6, which indicates that the system changes from a periodic state into a chaotic state when $k=-77.9$.

When $k$ is approximately -77.54 , the tangent bifurcation occurs, leading to an intermittent chaos and forming the period- 3 windows and then three stable periodic trajectories.

The period doubling bifurcations occur for the three trajectories when $k$ is approximately $-77,-76.16$, or -75.5 . When $k$ is approximately -74.6 , the secondary chaotic band and the unstable periodic trajectories converge into chaos.

When $k$ is approximately -73.58 , the tangent bifurcation occurs. The system suddenly switches from chaotic state to periodic state and the period-three windows forms. With the occurrence of a series of period doubling bifurcation, the system enters into the chaotic state again when $k$ is between -71.18 and -52.64 , upon which the system switches back from the chaotic state to the periodic state.

\section{Movement Characteristic Analysis of the Underwater Supercavitating Vehicle}

When the system of the supercavitating vehicle is not controlled, the movement state of the system is unstable $[12,14]$. To investigate its movement characteristics alone, according to the two-dimensional bifurcation diagram, the rest of the parameters of the system should be kept constant. Assuming that $\sigma$ is equal to 0.0315 , the feedback control laws $\delta_{c}=$ $15 z-30 \theta-0.3 q$ and $\delta_{e}=-21.95 z$, which corresponds to the point $(0.0315,-21.95)$ in the red stable movement area in Figure 2. The phase trajectory diagram is shown in Figure 7(a) when the control gain of the fin deflection angle $k$ is equal to -21.95 .

It can be observed that when $k=-21.95$, the phase trajectory of the system gradually stabilizes at an equilibrium point. The Lyapunov exponent spectrum as a function of time is presented in Figure 7(b) in which the values of the largest Lyapunov exponent curve are negative within a finite time.

The motion state of the supercavitating vehicle is presented in Figure 8, in which the system state variables, namely, the vertical position $z$, the transverse speed $w$, the pitch angle $\theta$, and the pitch rate $q$, are attracted to the equilibrium point $(0.0047,0.0866,0.0012,0)$ with less settling time under the control of the law of stable movement.

Figures $8(\mathrm{e})$ and $8(\mathrm{f})$ demonstrate that the immersion depth $h^{\prime}$ of the fin and the corresponding planing force $F_{p}$ are both 0 . This indicates that the fin is inside the cavity and does not have any contact with the cavity; the vehicle is in a stable navigation state.

When $\sigma$ is equal to 0.0315 , the feedback control laws are $\delta_{c}=15 z-30 \theta-0.3 q$ and $\delta_{e}=3 z$, which corresponds to the point $(0.0315,3)$ in the green periodic oscillation region in Figure 2.

Figure 9(a) tells us that the phase trajectory is a limit cycle with period 2 when the control gain of the fin deflection angle $k$ is equal to 3 . The Lyapunov exponent spectrum corresponding to $k=3$ is shown in Figure 9(b). It is not difficult to find that the system approximately has a zero Lyapunov exponent and three negative Lyapunov exponents.

The motion state of the supercavitating vehicle is shown in Figure 10, in which the system state variables, namely, the vertical position $z$, the transverse speed $w$, the pitch angle $\theta$, and the pitch rate velocity $q$, oscillate periodically at the equilibrium point $(0.0416,1.3937,0.0190,0)$.

The immersion depth $h^{\prime}$ of the fin oscillates periodically in the range of $[0,0.06]$ (in $\mathrm{m}$ ), which indicates that the vehicle continuously collides with the cavity wall.

The fin is inside the cavity at times and does not come in contact with the cavity, which results in zero planing force $F_{p}$. The fin penetrates the cavity into the water at times and produces the planing force oscillating periodically in the range of $[0,48]$ (in $\mathrm{N}$ ). The above actions repeat again and again, and such phenomenon is referred to as "fin attack phenomenon." It also indicates that the vehicle is in an unstable periodic oscillating state. 


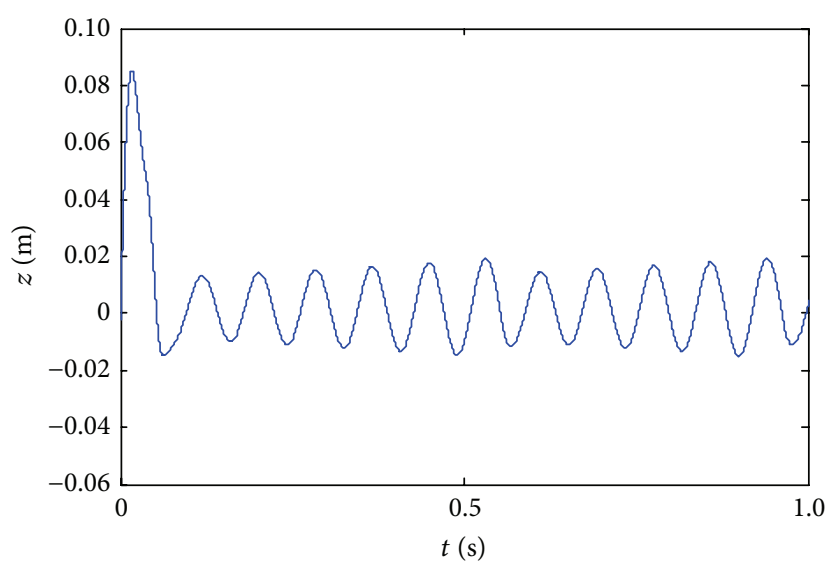

(a) Vertical position

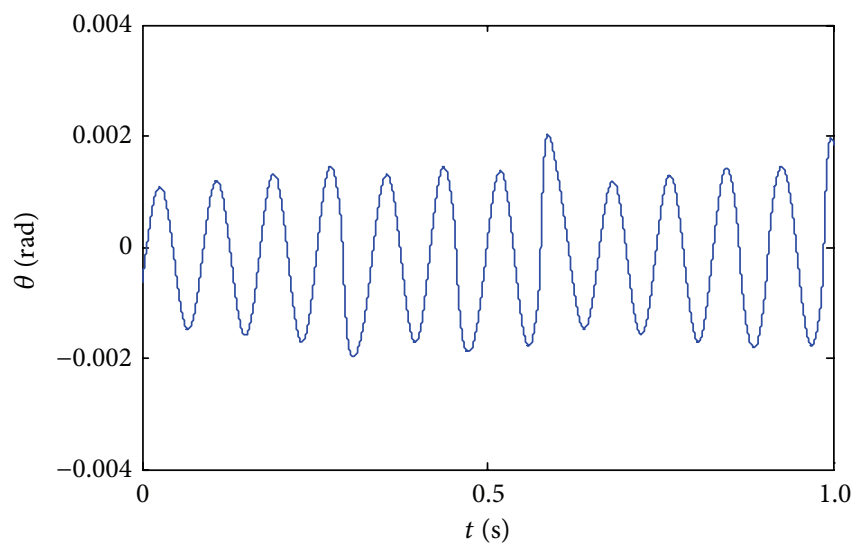

(c) Pitch angle

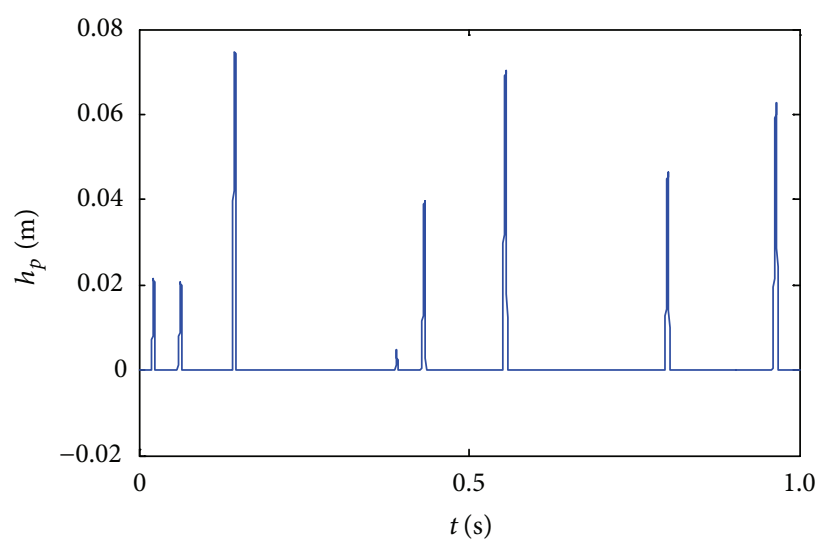

(e) Immersion depth

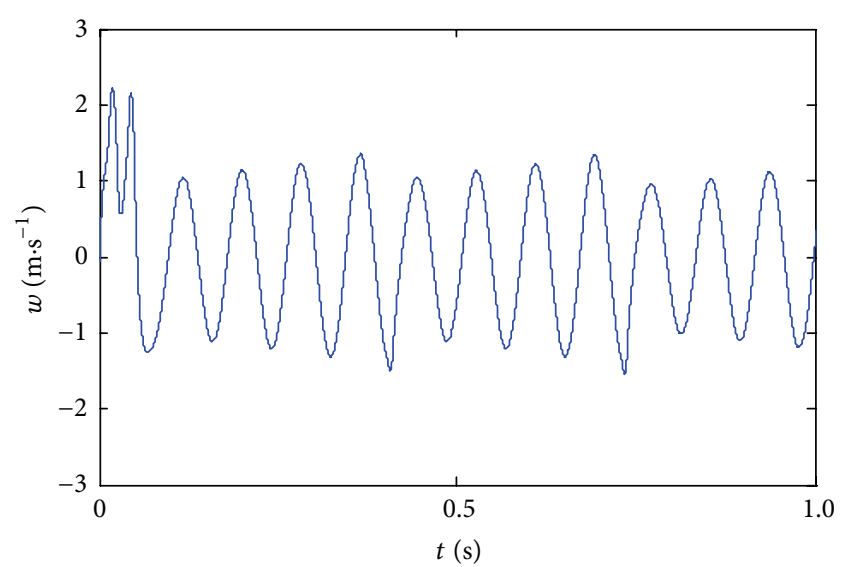

(b) Transverse speed

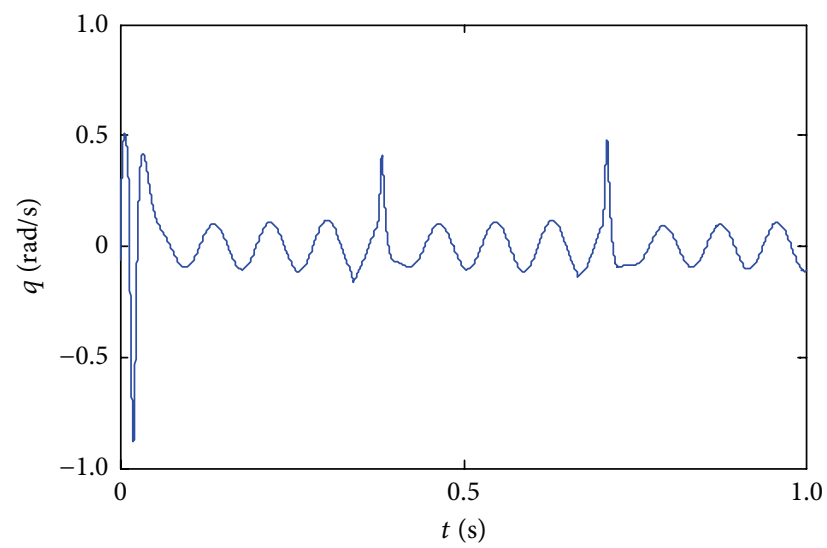

(d) Pitch rate

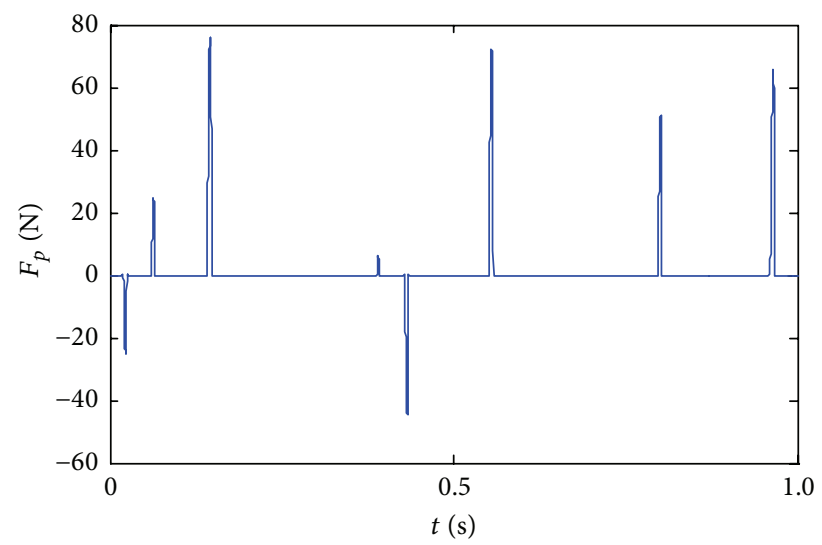

(f) Planing force

FIGURE 12: The motion state of the system when $k=55$.

When $\sigma$ is equal to 0.0315 , the feedback control laws of $\delta_{c}=15 z-30 \theta-0.3 q$ and $\delta_{e}=-55 z$ are selected, which corresponds to the point $(0.0315,-55)$ in the yellow chaotic area in Figure 2.

Figure 11(a) demonstrates that the phase trajectory is a chaotic attractor when the control gain of the fin deflection angle $k$ is equal to -55 , which indicates that the chaos has occurred and the movement of the vehicle has the characteristics of a nonlinear dynamic behavior. The Lyapunov exponent spectrum corresponding to $k=-55$ is given in Figure 11(b). It is relatively easy to find that the system has a positive Lyapunov exponent and three negative Lyapunov exponents, suggesting that the system is in a fourdimensional chaotic state.

The motion state of the system is presented in Figure 12. After the launch of the supercavitating vehicle, the four 
system state variables, namely, $z, w, \theta$, and $q$, are in the intense nonperiodic oscillating state. Thus, the vehicle in motion will experience instability.

It can be observed from Figures 12(e) and 12(f) that within the time range of $[1,2]$ (values in $s$ ), the fin of the vehicle is continuously into contact with the cavity wall under the action of gravity and produces the planing force. The planing force increases gradually with the increase of immersion depth and the vehicle will rebound inside the cavity, which results in the loss of the planing force. The above actions repeat subsequently and the planing force oscillates. The existence of the planing force will cause vibration and impact to the vehicle, resulting in the loss of stability of the vehicle. Therefore, precise control must be exerted on the vehicle to avoid the above situations $[16,17]$.

\section{Conclusions}

The nonlinear dynamic characteristic movement states under different control parameters of the supercavitating vehicle were analyzed based on a four-dimensional dynamical model of the vehicle. The following conclusions have been mainly derived:

(1) The movement trajectories of the supercavitating vehicle have complicated dynamical behavior: the system will experience Hopf bifurcation, periodical windows, chaos, and other nonlinear phenomena when the control parameters vary.

(2) The movement state of the vehicle under different control parameters was numerically and precisely analyzed according to the phase trajectory diagram, the bifurcation diagram, and the Lyapunov exponential spectrum.

(3) Most importantly, the authors were the first to find that the range of parameters of the vehicle in any movement state can be determined by a twodimensional bifurcation diagram. The importance of selecting appropriate control parameters to realize the stable navigation of the supercavitating vehicle was demonstrated.

It is believed that the work presented in this paper is of great importance for further studies on the stable control of the underwater supercavitating vehicles, especially for engineering practice.

\section{Competing Interests}

The authors declare that there is no conflict of interests regarding the publication of this paper.

\section{Acknowledgments}

This work is supported by the National Natural Science Foundation of China (nos. 11472163, 11402116, and 61473340).

\section{References}

[1] G. Wang and M. Ostoja-Starzewski, "Large eddy simulation of a sheet/cloud cavitation on a NACA0015 hydrofoil," Applied Mathematical Modelling, vol. 31, no. 3, pp. 417-447, 2007.

[2] Q. T. Li, Y. S. He, and L. P. Xue, "A numerical simulation of pitching motion of the ventilated supercaviting vehicle approximately its front," Chinese Journal of Hydrodynamics, vol. 26, no. 6, pp. 589-685, 2011.

[3] A. K. Singhal, M. M. Athavale, H. Li, and Y. Jiang, "Mathematical basis and validation of the full cavitation model," Journal of Fluids Engineering, vol. 124, no. 3, pp. 617-624, 2002.

[4] Y. N. Savchenko, "Supercavitation problems and perspectives," in Proceedings of the 4th International Symposium on Cavitation, Pasadena, Calif, USA, April 2001.

[5] M. A. Hassouneh and E. H. Abed, "Lyapunov and LMI analysis and feedback control of border collision bifurcations," Nonlinear Dynamics, vol. 50, no. 3, pp. 373-386, 2007.

[6] Y. J. Wei, J. H. Wang, J. Z. Zhang, W. Cao, and W. H. Huang, "Nonlinear dynamics and control of underwater supercavitating vehicle," Journal of Vibration And Shock, vol. 28, no. 6, pp. 179-204, 2009.

[7] S. S. Kulkarni and R. Pratap, "Studies on the dynamics of a supercavitating projectile," Applied Mathematical Modelling, vol. 24, no. 2, pp. 113-129, 2000.

[8] J.-Y. Choi, M. Ruzzene, and O. A. Bauchau, "Dynamic analysis of flexible supercavitating vehicles using modal-based elements," Simulation, vol. 80, no. 11, pp. 619-633, 2004.

[9] B. Feeny, "A nonsmooth Coulomb friction oscillator," Physica D. Nonlinear Phenomena, vol. 59, no. 1-3, pp. 25-38, 1992.

[10] A. D. Vasin and E. V. Paryshev, "Immersion of cylinder in a fluid through a cylindrical free surface," Fluid Dynamics, vol. 36, no. 2, pp. 169-177, 2001.

[11] G. Lin, B. Balachandran, and E. Abed, "Supercavitating body dynamics, bifurcations and control," in Proceedings of the American Control Conference (ACC '05), pp. 691-696, Portland, Ore, USA, June 2005.

[12] J. Dzielski and A. Kurdila, "A benchmark control problem for supercavitating vehicles and an initial investigation of solutions," Journal of Vibration \& Control, vol. 9, no. 7, pp. 791804, 2003.

[13] G. J. Lin, B. Balakumar, and H. A. Eysd, "Bifurcation behavior of a supercavitating vehicle," in Proceedings of the ASME International Mechanical Engineering Congress and Exposition, pp. 293-300, Chicago, Ill, USA, November 2006.

[14] G. J. Lin, B. Balachandran, and E. H. Abed, "Dynamics and control of supercavitating vehicles," Journal of Dynamic Systems, Measurement and Control, vol. 130, no. 2, Article ID 021003, pp. 281-287, 2008.

[15] M. A. Hassouneh, V. Nguyen, B. Balachandran, and E. H. Abed, "Stability analysis and control of supercavitating vehicles with advection delay," Journal of Computational and Nonlinear Dynamics, vol. 8, no. 2, Article ID 021003, 2012.

[16] G. Lin, B. Balachandran, and E. H. Abed, "Nonlinear dynamics and bifurcations of a supercavitating vehicle," IEEE Journal of Oceanic Engineering, vol. 32, no. 4, pp. 753-761, 2007.

[17] J. H. Wang, Y. J. Wei, and K. P. Yu, "Modeling and control of underwater supercavitating vehicle based on memory effect of cavity," Journal of Vibration And Shock, vol. 29, no. 8, pp. 160163, 2010. 


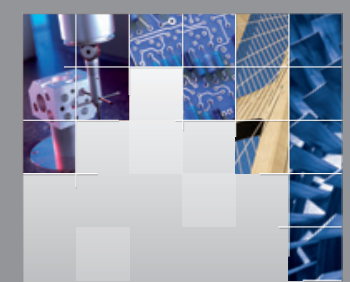

\section{Enfincering}
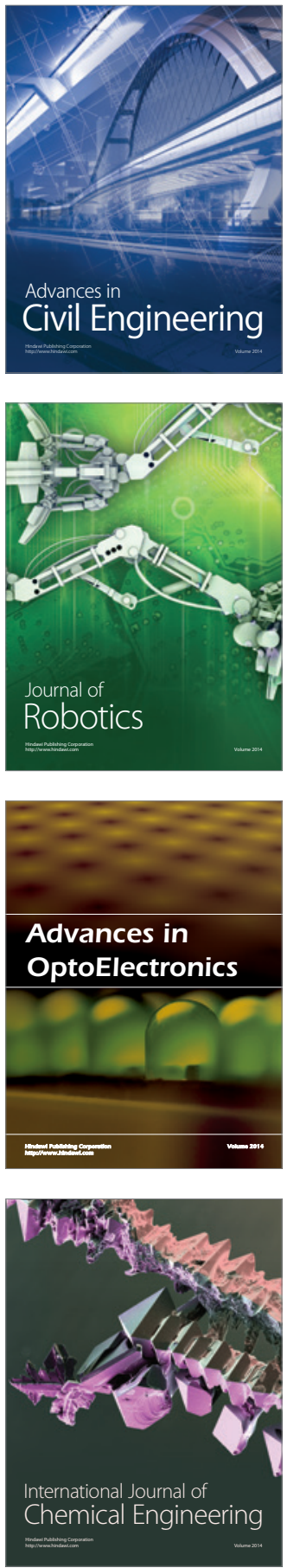

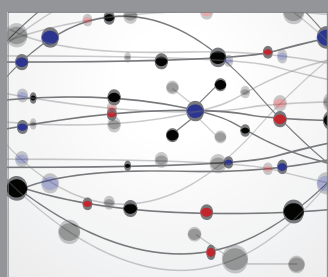

The Scientific World Journal

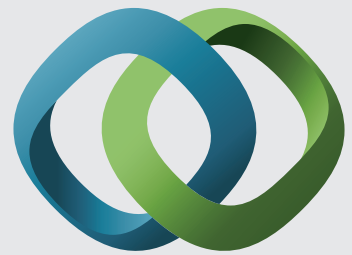

\section{Hindawi}

Submit your manuscripts at

http://www.hindawi.com
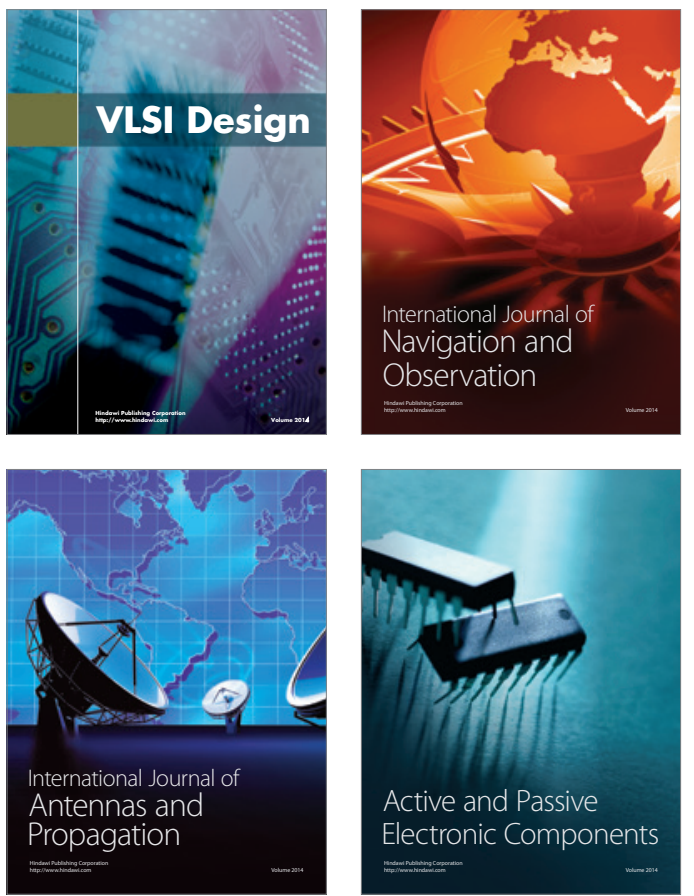
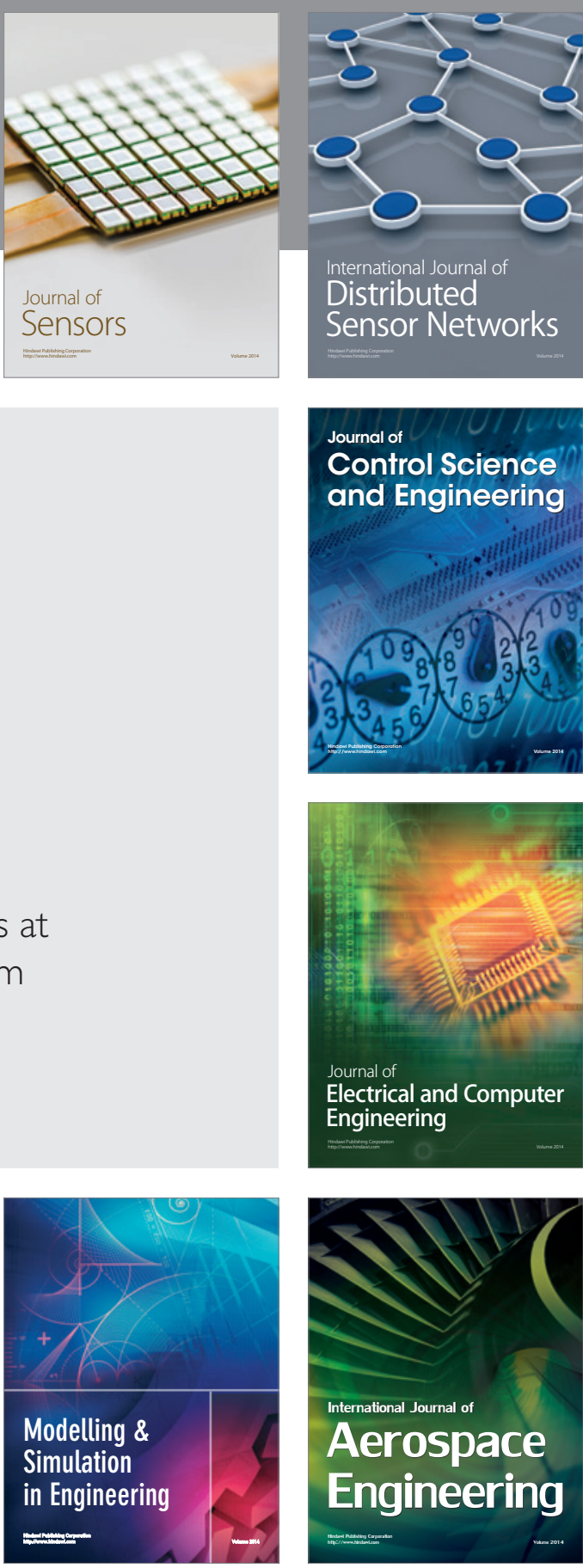

International Journal of

Distributed

Sensor Networks

Journal of

Control Science

and Engineering
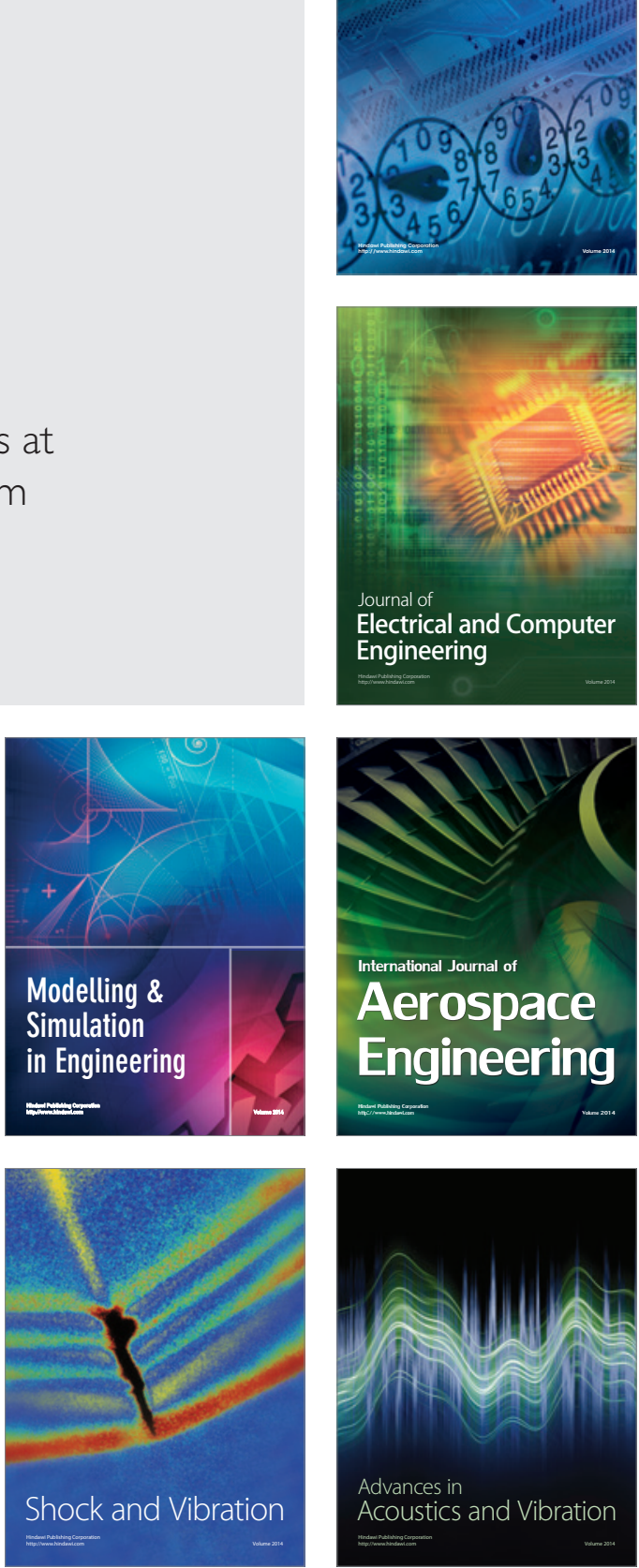